\title{
ANALISE DAS VARIAÇÕES DO COEFICIENTE DE ATRITO E AS CORRELAÇÕES COM OS MECANISMOS DE DESGASTE
}

\author{
E.M. Pantaleón', D. K. Tanaka ${ }^{2}$ e F.G. Bernardes ${ }^{2}$ \\ ${ }^{1}$ Grupo de Estudo de Tribologia - Universidade Federal do Rio Grande do Norte \\ ${ }^{2}$ Laboratório de Fenômenos de Superfícies - Universidade de São Paulo \\ epantaleon@ect.ufrn.br - dktanaka@usp.br
}

Artigo submetido em outubro/2011 e aceito em março/2012

\section{RESUMO}

Durante os ensaios de desgaste, os valores do coeficiente de atrito variam de acordo com as diferentes condições dos sistemas tribológicos associados a parâmetros como carga, velocidade e temperatura de contato. As variações complexas do coeficiente de atrito dificultam uma correlação com as medidas do sinal de desgaste. Neste trabalho, foi desenvolvido um método para a análise de desgaste usando técnicas estatísticas,

que analisou o sinal do coeficiente de atrito. A partir de um teste de pino/disco dinâmico, verificaram-se diferentes tipos de mecanismos de desgaste sob diferentes condições de carga. A análise proposta permite a representação dos mecanismos de desgaste que atuam nos materiais em forma de mapa, para correlacionar às variações dos desvios-padrão do coeficiente de atrito com a taxa de desgaste.

PALAVRAS-CHAVE: desgaste, tribologia, mecanismos de desgaste, mapa de desgaste

\section{ANALYSIS OF THE VARIATIONS OF THE COEFFICIENT OF FRICTION AND THE CORRELATIONS WITH THE WEAR MECHANISMS}

\begin{abstract}
During the wear tests, the friction coefficient value varies according to the different conditions of tribological systems associated with parameters such as load, speed and contact temperature. The complex variations of friction coefficient make difficult a correlation with the measures of the signs of wear. In this paper, a method was developed for the analysis of wear using statistical techniques, which looked at the
\end{abstract}

sign of the friction coefficient. From a dynamic test pin/disk, there are different types of wear mechanisms under different loading conditions. The proposed analysis allows the representation of the wear mechanisms that operate in the materials in form of a map to correlate the changes in standard deviations of the friction coefficient on the wear rate.

KEY-WORDS: wear, tribology, wear mechanism, wear map. 


\section{ANALISE DAS VARIAÇÕES DO COEFICIENTE DE ATRITO E AS CORRELAÇÕES COM OS MECANISMOS DE DESGASTE}

\section{INTRODUÇÃO}

A necessidade de controlar o atrito e o desgaste em terra, mar e sistemas de propulsão aeroespacial foi claramente o impulso histórico para investir nas pesquisas, que correlacionava o coeficiente de atrito ao desgaste (BLAU, 1997). As primeiras pesquisas sobre o atrito e desgaste ocorreram entre 1940 e 1950 realizadas por engenheiros mecânicos e metalurgistas, para obter dados para ajudar na construção de motores, freio, rolamentos, e outros tipos de movimentos mecânicos.

A história do desgaste por deslizamento em metais não pode ser separada dos estudos clássicos de atrito, pois ambas envolvem mecanismos dos corpos sólidos em contacto. $\mathrm{O}$ atrito e o desgaste foram motivo de estudo por Terheci et al (1995), que descreveram a correlação entre as seqüências dos mecanismos que se geram nos complexos fenômenos de desgaste e atrito como: aderência, delaminação, a transição nos contatos de dois a três corpos e deformações plásticas e elásticas, quebras, debris entre outros.

Pantaleón (2004), afirma que as transições em mecanismos de desgaste dependem do sistema tribologico, mais do que as características dos materiais o que torna complexo um arranjo experimental sistemático do estudo. Além disso, observa que existem dificuldades em prever as ocorrências das transições dos mecanismos de desgaste, porque abrangem muitas variáveis que promovem as alterações das propriedades tanto topográficas e físico-químicas das superfícies de contacto dos materiais.

O crescente avanço da ciência da computação em 1980 teve grande influência sobre todos os campos de aplicação incluindo o sensoriamento e monitoração do desgaste (CALLACOT, 1977). A vibração induzida nas superfícies em contacto pelo atrito depende de uma combinação dos materiais em contacto e dos parâmetros experimentais como carga, velocidade e principalmente, das características do sistema de ensaio com sua freqüência natural e inércia dos equipamentos e componentes (ARNOV et al., 1984 e KRISHNA et at., 1997).

Para a construção de mapas de desgaste, é necessária a realização de grande quantidade de ensaios, de forma geral, demorados. Por isso, os mapas reportados na literatura, Lim e Ashby (1987), Quinn (1992) e Blau (1997), em geral, reúnem resultados de vários pesquisadores, embora a literatura tenha mostrado que os resultados apresentam, grande dispersão, quando não são realizados no mesmo equipamento e com condições extremamente próximas.

Pantaleón e Dos Santo Lima (2011), aplicam em seu trabalho o conceito de transformada wavelet, como uma ferramenta útil e robusta matematicamente para detectar transições de mecanismos de desgaste usando as informações do coeficiente de atrito e proporcionando uma base poderosa $\mathrm{n}$ a detecção das mudanças nas freqüências do sinal.

Neste trabalho, temos como hipóteses que as variações dos desvios padrões dos parâmetros tribológicos, contêm informações dos mecanismos de desgastes que acontecem 
ao longo do ensaio, pelo qual o principal objetivo é correlacionar as variações do desgaste que acontecem em um ensaio pino sobre disco, com as variações do coeficiente de atrito, usando os métodos estatísticos tradicionais para o analise dos dados obtidos do tribosistema estudado. O uso da programação em MATLAB 6,5 como ferramenta auxiliar na pesquisa, permitiu a obtenção da correlação existente entre os parâmetros tribológicos.

\section{PROCEDIMENTO EXPERIMENTAL}

Para o estudo do desgaste e do coeficiente de atrito foram realizados ensaios de pino/disco em uma máquina Plint \& Partners modelo TE-67 apresentado na figura 1 . As cargas normais utilizadas variaram de 20 a 40 N, 20 a $80 \mathrm{~N}$ e 20 a $120 \mathrm{~N}$, com uma velocidade de deslizamento de $1 \mathrm{~m} / \mathrm{s}$, em 3 horas. Tanto os pinos e os discos foram lixados cuidadosamente com lixas d'água de carburo de silício (SiC) com granulação 1000 e posteriormente submergidos em banho de etanol em um limpador ultrasônico. No início de cada ensaio, foi programada uma etapa onde a carga normal é fixada em $15 \mathrm{~N}$, com uma velocidade de deslizamento de $0,8 \mathrm{~m} / \mathrm{s}$ durante 30 minutos.

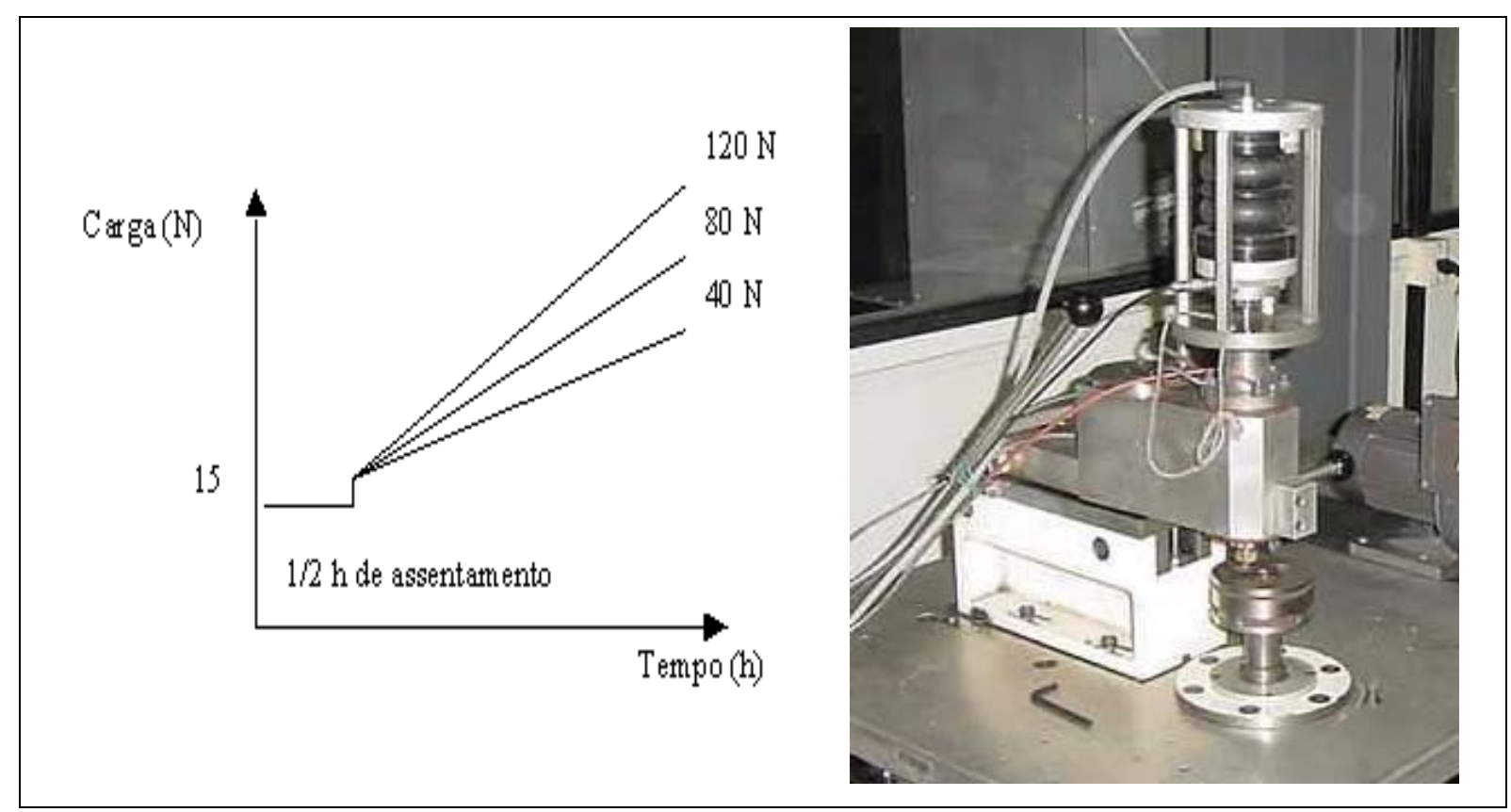

Figura 1 - Esquema dos ensaios dinâmicos em maquina pino sobre disco

Durante os ensaios, foi medida a força de atrito, a carga aplicada a distancia de deslizamento dos pinos sobre os discos, o potencial de contato (proporcional à resistividade elétrica do contato), o deslocamento vertical dos pinos (daqui diante "desgaste $(\mu \mathrm{m})$ " e a temperatura dos pinos).

\section{- Analise química}

Para a certificação dos materiais ensaiados foi realizadas as análise química usando o método convencional da via úmida para a medição dos elementos químicos típicos de aços ao carbono e ferramenta exceto o carbono (C) e o enxofre (S) que foram medidos pelo método 
gaso-volumétrico (VOGEL, 2002) e os resultados estão apresentados na tabela 1.

Tabela 1: Composição química das amostras de pino e disco.

\begin{tabular}{|l|l|l|l|l|l|l|l|l|l|}
\hline Materiais & $\mathbf{C ( \% )}$ & S(\%) & $\mathbf{M n}(\%)$ & Si(\%) & $\mathbf{C r}(\%)$ & Ni(\%) & Mo(\%) & W(\%) & V(\%) \\
\hline PINO (M2) & 0,79 & 0.02 & 0,24 & 0,24 & 4,25 & - & 0,35 & 6,65 & 1,97 \\
DISC(1045) & 0,44 & 0.02 & 0,52 & 0,17 & & & & & \\
\hline
\end{tabular}

- Material dos discos

O material dos discos foi aço AISI 1045 (diâmetro $30 \mathrm{~mm}$, altura $10 \mathrm{~mm}$ ) com tratamento térmico. O tratamento consistiu em manter os discos em um forno tipo mufla durante 1 hora e 30 minutos de permanência a uma temperatura de $830 \pm 10$ 드, seguidamente submergidos durante 2 horas em um forno com banho de sal de nitrato de potássio a temperatura de $370 \pm 5 \circ \mathrm{C}$, obtendo uma dureza Rockwell de $\mathrm{Rc}=40,6 \pm 0,5$. $\mathrm{A}$ microestrutura obtida depois do tratamento se apresenta na figura 2 (a).

- Material dos pinos

O material utilizado para os pinos (diâmetro $3 \mathrm{~mm}$, altura $6 \mathrm{~mm}$ ) foi do tipo AISI M2 (aço ferramenta). $O$ tratamento térmico consistiu primeiramente em dois pré-aquecimento as diferentes temperatura, a $550^{\circ} \mathrm{C}$ e $850^{\circ} \mathrm{C}$ durante 30 minutos de permanência em cada uma, seguidamente uma austenitização a 1.180 ㄷ C durante 1 hora e têmpera ao ar, seguido de três revenidos em banho de sal, de 2 horas de permanência para cada um, a uma temperatura de 550 ㄷ $\mathrm{C}$ obtendo-se a dureza $\mathrm{Rc}=64,8 \pm 0,44$. A microestrutura obtida depois do tratamento se apresenta na figura 2 (b).

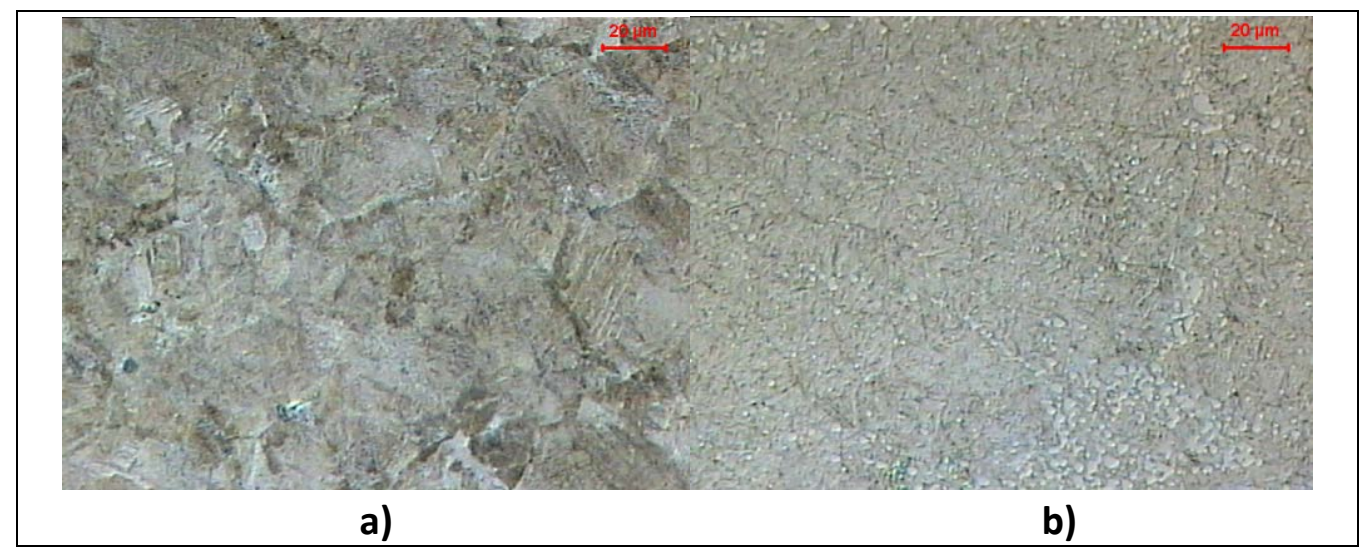

Figura 2 - Metalografia dos Materiais estudados. 500x. Atacado com Nital 2\%.

a) Disco: Microestrutura bainitica. b) Pino: Microestrutura de martensita revenida com presença de carbonetos.

\section{ANALISE DOS DADOS}

As flutuações do coeficiente de atrito estão associadas a fatores como desgaste, quebra de óxidos, à variação da carga, velocidade e a fenômenos associados com a remoção de debris, assim como das vibrações transmitidas das partes dos componentes móveis do equipamento ou do ambiente, etc. A complexa variação do sinal do coeficiente de atrito por 
fatores que influenciam indiretamente na referida medida, dificultam o entendimento do papel deste parâmetro no mecanismo de desgaste, daí que a utilização de técnicas estatísticas é um intento de poder contribuir para resolver estes problemas (PANTALEON, 2004 ).

O desvio padrão (STD) e a média (Md) dos parâmetros tribológicos medidos são valores que contêm informação sobre o processo que ocorrer durante o ensaio. Estes dois valores podem ser calculados pelas equações 1 e 2 , respectivamente.

$$
M d=\frac{\sum_{i=1}^{n} P(i)}{n}
$$

$$
S T D=\sqrt{\frac{\sum_{i=1}^{n}\left(P(i)-P_{o}\right)^{2}}{n}}
$$

equação (2)

Os valores experimentais destes parâmetros tendem a variar com o desgaste, precisando analisar as variações dos desvios padrões e medias móveis dos sinais ( $\triangle S T D$ e $\Delta M d$ ), onde $i$ corresponde aos números dos intervalos dos estudos, nos quais são agrupados os conjuntos de valores das magnitudes medidas. Cada conjunto de valores contem 15 dados e o modelo matemático das diferenças e apresentados nas equações 3 e 4 . Para viabilizar os cálculos foi elaborado um programa em Matlab 6.5 como ferramenta para obter os comportamentos móveis das medias e dos desvios padrões dos parâmetros medidos nos ensaios de pino sobre disco.
$\triangle S T D(i)=S T D(i+1)-S T D(i)$
equação (3)
$\Delta M d(i)=M d(i+1)-M d(i)$
equação (4)

\section{ANALISE DOS RESULTADOS}

\section{- Resultado dos ensaios pino sobre disco}

A figura 3 mostra as variações no tempo dos parâmetros do ensaio como desgaste (posição do pino no disco), o potencial de contato, força de atrito e coeficiente de atrito. As diferentes cores representam cada intervalo dos testes. A cor azul representa intervalos de cargas de 20 a $120 \mathrm{~N}$; verde de 20 a $80 \mathrm{~N}$ e o vermelho de 20 a $40 \mathrm{~N}$. Os diagramas do coeficiente de atrito, e do desgaste indicam que devem existir diferentes transições no tempo de ensaio. $O$ primeiro mecanismo pudesse associar quando acontecem as maiores flutuações dos parâmetros.

Analisando os valores da força de atrito, se observa que todas as curvas se comportaram de maneira crescente durante todo o período do ensaio com o aumento progressivo da carga normal aplicada. Porém, no coeficiente de atrito não se nota uma relação aparente com a carga aplicada, e o comportamento em função do tempo foi distinto para cada tipo de carregamento. 
Quanto aos resultados de desgaste, observa-se uma evolução crescente em função do tempo de ensaio para os três tipos de carregamento. Nota-se um elevado aumento do desgaste, logo nos instantes iniciais dos ensaios com as faixas de carregamentos menores.

As maiores perdas ocorreram para as menores faixas de variação de carga (curva verde e vermelha). Esse fato poderia ser explicado pela ocorrência do processo de oxidação nas superfícies ser predominante no ensaio com a maior variação da carga, o qual tendeu a diminuir a perda de material nessa condição (QUINN, 1962, SO, 1995 e PANTALEON, 2004).

Quanto ao comportamento do potencial de contato, as curvas mostradas na figura 3, indicam que não houve uma dependência característica do potencial com a evolução da carga durante o ensaio. Por outro lado, os maiores valores evidenciados no ensaio, com a maior variação de carga (curva azul), podem também estar relacionados com a ocorrência de um filme de óxido que teria provocado um isolamento das superfícies de contato com esse tipo de carregamento. Esse fato concorda com o resultado correspondente ao desgaste, entretanto a observação do coeficiente de atrito, correspondente à curva azul, indica que se o filme de óxido existiu, o mesmo não afetou o valor médio do coeficiente de atrito de maneira significativa.

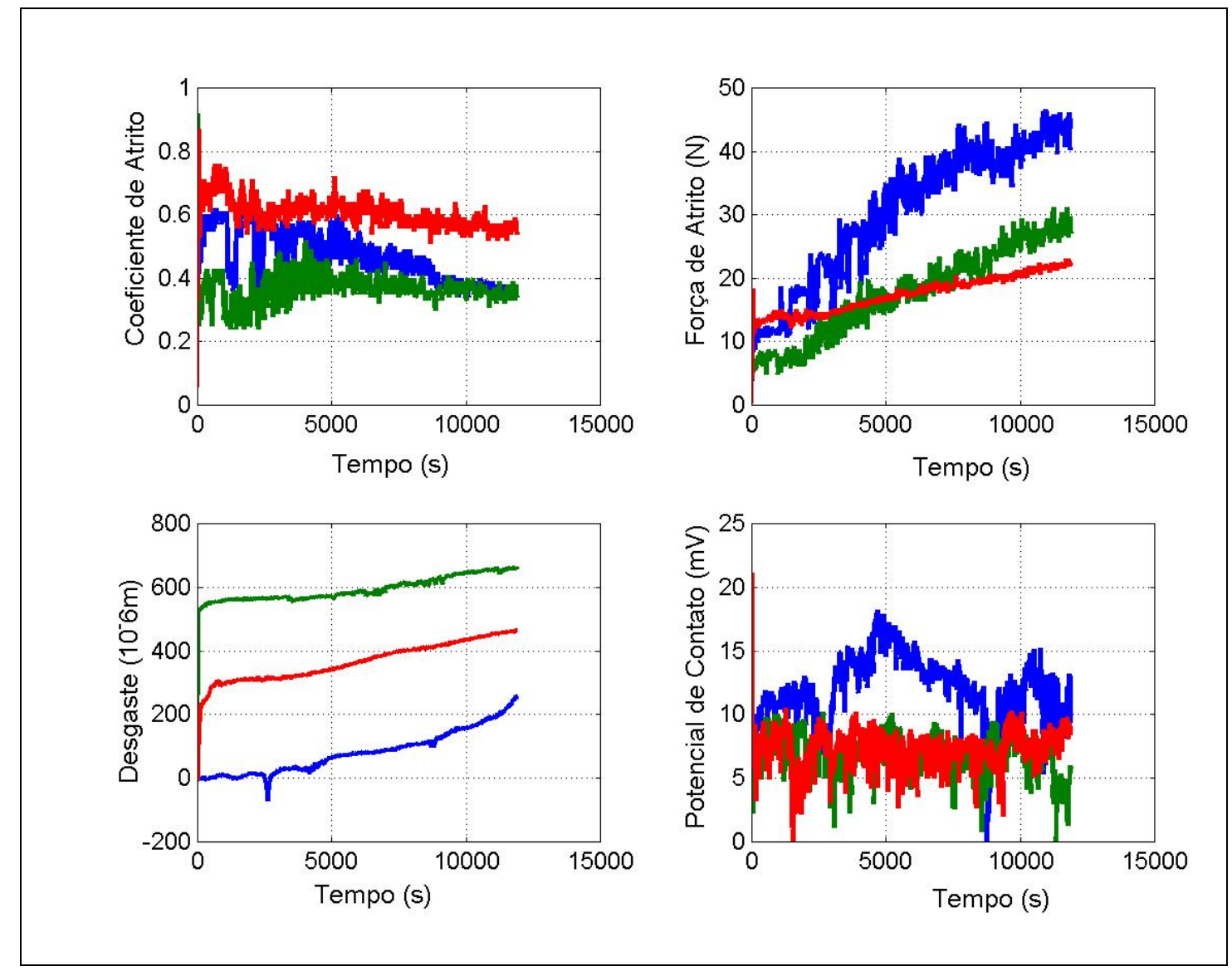

Figura 3 - Sinais reportados de maquina Standard pino sobre disco Força de Atrito, desgaste, coeficiente de atrito e potencial de contato. Azul ( 20 a $120 \mathrm{~N}$ ), Verde (20 a $80 \mathrm{~N})$ e Vermelho (20 a $40 \mathrm{~N})$. 
Também se pode observar que, no ensaio com a maior variação da carga (curvas azuis), as flutuações na amplitude dos valores das curvas de todos os parâmetros foram significativamente maiores. Esse fato poderia estar associado à maior instabilidade do sistema nessa condição de ensaio, porém, existe a hipótese de que as flutuações, e mesmo as suas variações, possam estar associadas aos fenômenos característicos de cada faixa de variação de carga (PANTALEON, 2004).

\section{- Resultados da técnica estatística}

Considerando que os resultados obtidos na máquina de ensaio são sinais aleatórios no tempo, podemos aplicar os conceitos estatísticos, onde se define que uma variável aleatória é constante se seus momentos estatísticos são constantes. No caso dos ensaios, vamos considerar a hipóteses, que se as variações das médias e dos desvios-padrões dos parâmetros tribológicos são constantes em certos instantes, então o "processo" correspondente, ou seja, os fenômenos referentes ao parâmetro analisado (coeficiente de atrito, potencial de contato ou desgaste) se torna estável nesses pontos.

Nas figuras 4 e 5 apresentam-se respectivamente as variações moveis das médias $(\triangle M d$ ) e dos desvios-padrões $(\triangle S T D)$ de cada parâmetro tribológico resultantes dos diferentes tipos de ensaio. A variação da média do coeficiente de atrito, como pode ser observada, em um determinado instante passa a ser constante. Nos primeiros $3.000 \mathrm{~s}$, a variação da média dos parâmetros de ensaios apresentam valores que decrescem, tendo o mesmo comportamento para as diferentes cargas de ensaio, ou seja, podemos prever uma convergência das tendências dos valores medidos, e assumir que são os instantes onde as superfícies em contatos adquirem maior conformidade entre elas. Considerando que a variação passou a ser constante ou nula depois de $3.000 \mathrm{~s}$, se deduz que um processo estável do coeficiente de atrito prevalece e podemos assumir que um mecanismo de desgaste é privilegiado. (PANTALEÓN \& DOS SANTO LIMA, 2011).

A variação da média do potencial de contato se dá com maiores oscilações e permanece além dos $3.000 \mathrm{~s}$, mas ao final do ensaio, sua variação torna-se constante. Os instantes com instabilidade da variação da média do potencial indicam que os mecanismos existentes no contato permaneceram instáveis quanto às características elétricas, podendo indicar que existem desprendimentos ou degeneração das superfícies que provocam instabilidade nos processos.

A variação da média $(\Delta M d)$ do desgaste tendeu a constante, em tempos menores do que para o coeficiente de atrito e para o potencial de contato, o que permite deduzir que um mesmo mecanismo de desgaste pode ter valores médios diferentes do coeficiente de atrito. 

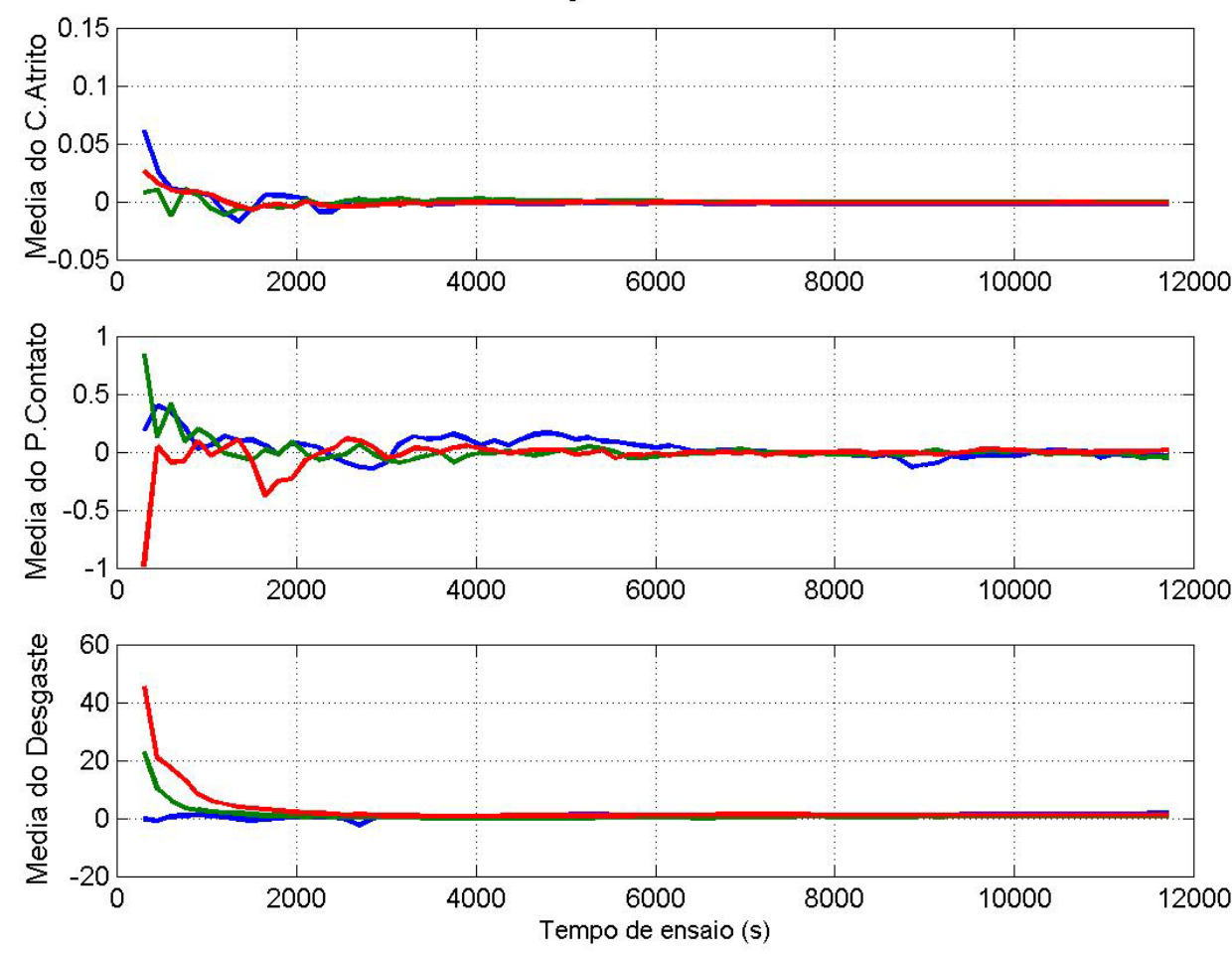

Figura 4- Variação da media do coeficiente de atrito, potencial de contato e do desgaste. Azul ( 20 a $120 \mathrm{~N}$ ), Verde (20 a 80N) e Vermelho (20 a $40 \mathrm{~N}$ ).
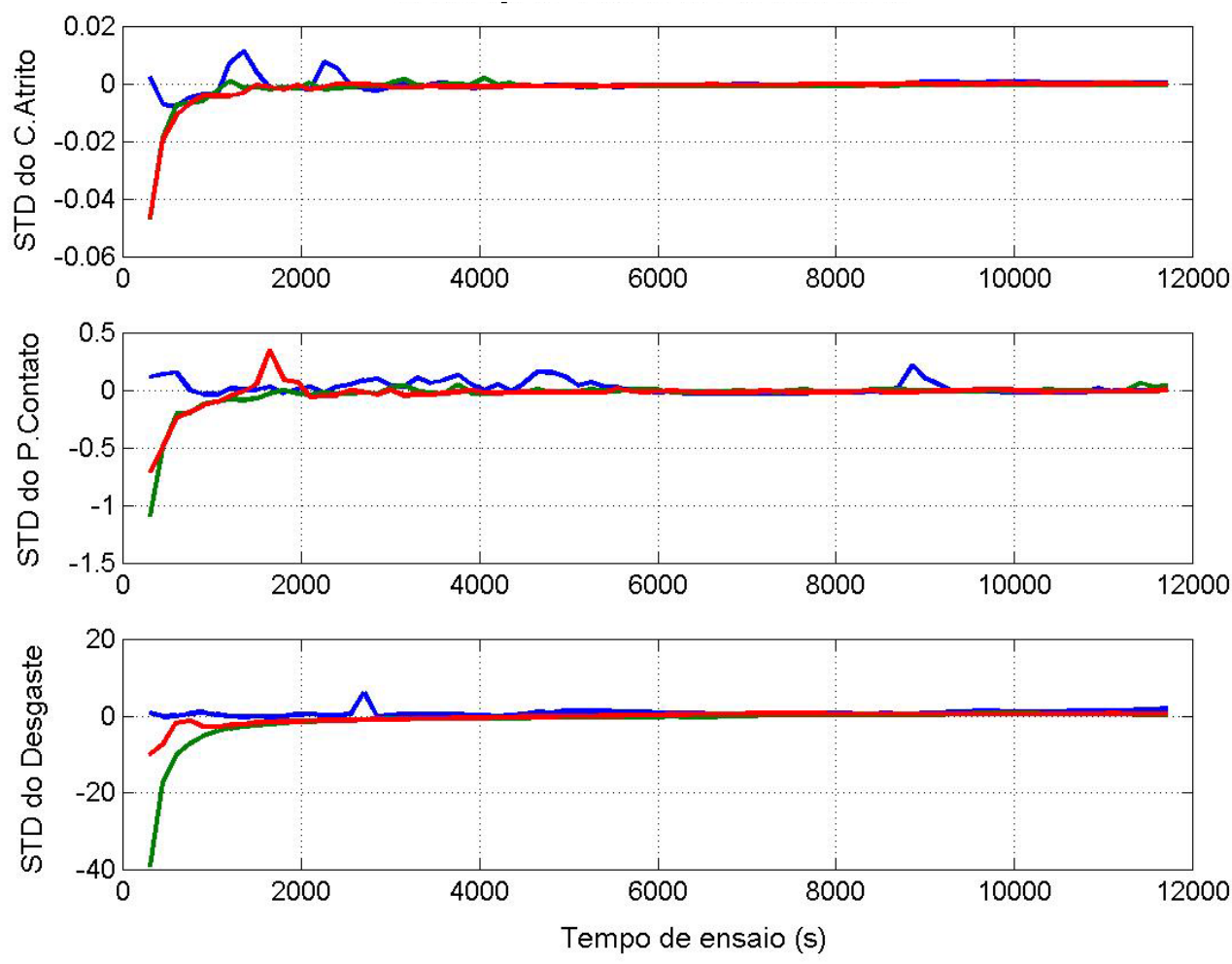

Figura 5 - Variação do Desvio Padrão do coeficiente de atrito, potencial de contato e do desgaste. Azul ( 20 a 120 N), Verde (20 a $80 N$ ) e Vermelho (20 a 40 N). 


\section{- Construção de mapa de desgaste}

A figura 6 apresenta em um gráfico a clusterização de todos os resultados dos ensaios, com os valores das variações dos desvios padrões dos parâmetros de desgaste e coeficiente de atrito, permitindo a separação de regiões tribológicas com diferentes características. Este tipo de apresentação de duas dimensões é função de outros parâmetros, uma vez que o coeficiente de atrito depende da temperatura de contato, velocidade, carga aplicada, etc (LIM \& ASHBY, 1987 e PEZDIRNIK et al., 1999). Os valores do desvio-padrão do coeficiente de atrito que apresentam a maior taxa de desgaste são os pontos correspondentes ao running-in evidenciados na figura $7(a)$, que são a característica de um mecanismo de desgaste severo. Os pontos em menores valores do desvio-padrão do coeficiente de atrito correspondem ao mecanismo de desgaste oxidativo como mostra a figura 7(b). O mecanismo de desgaste adesivo, não foi constatado durante os testes, mas poderia estar presente durante running-in, e pode ser presumido na região onde acontecem altas taxas de desgaste, com pequenos valores do desvio do coeficiente de atrito.

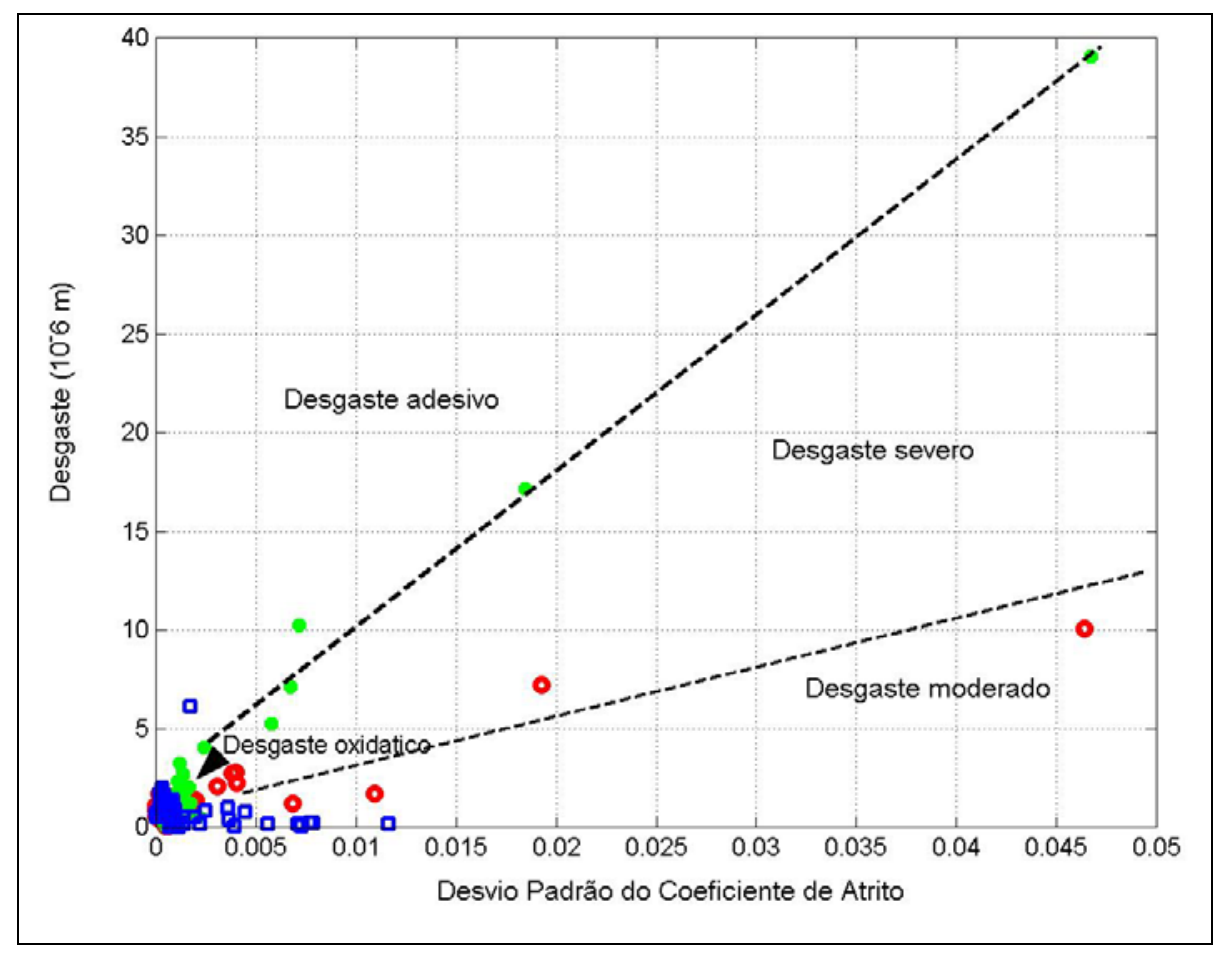

Figura 6 - Mapa de desgaste partindo das variações dos desvios padrões do coeficiente de atrito e do desgaste. As cores representam os intervalos dos ensaios.

O estudo das superfícies ensaiadas foi usando um microscópio eletrônico de varredura (SEM), principalmente nos períodos onde a variação dos desvios padrões móvel do coeficiente de atrito era estável. A figura 8(a) mostra áreas com camadas de óxido que estão sobre a superfície e se apresentam em formas de laminas com fissuras. As superfícies com laminas de óxidos com fissuras foram observadas nos ensaios com variações de carga de 20 a $40 \mathrm{~N}$ e de 20 a $80 \mathrm{~N}$. Para os ensaios com cargas até $120 \mathrm{~N}$, as camadas tem uma morfologia diferente com óxidos globulares como apresentado na figura 8(b). A presença destes tipos de camadas permite diferenciar dos tipos de desgaste oxidativo, sendo considerado moderado o 
correspondente as laminas com rachaduras, e severo, onde prevalecem a morfologia globular (QUINN, 1962 e HO, 1995).

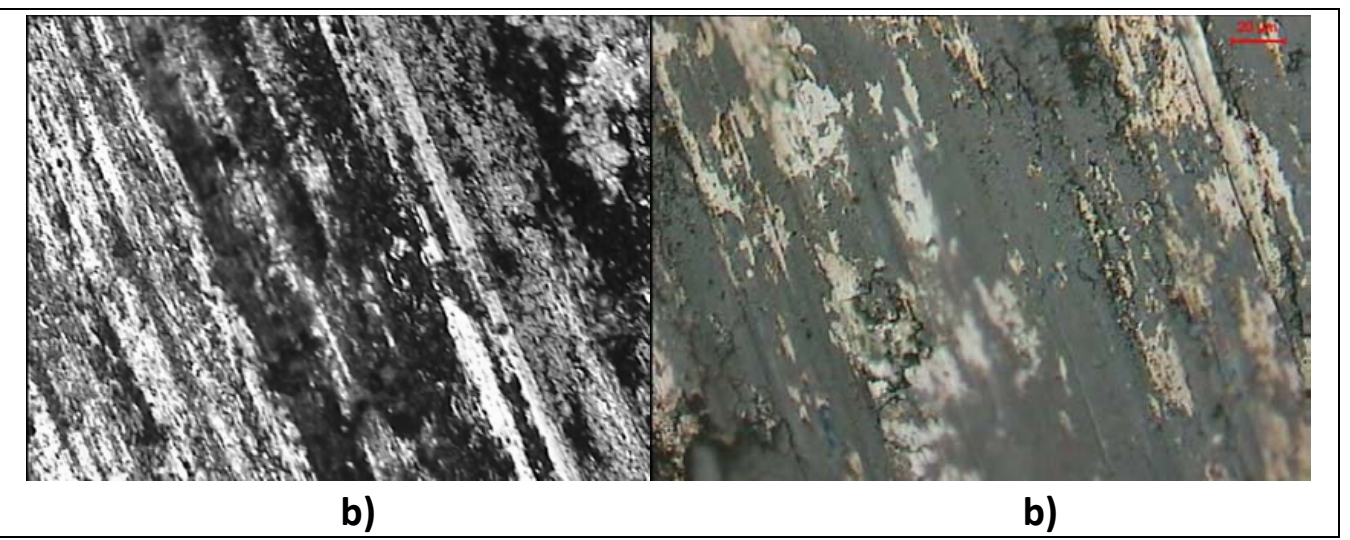

Figura 7 - Morfologia das superfícies analisadas em um microscópio óptico.

a) running-in: superfície riscada. b) laminas trincada de filmes de óxidos.

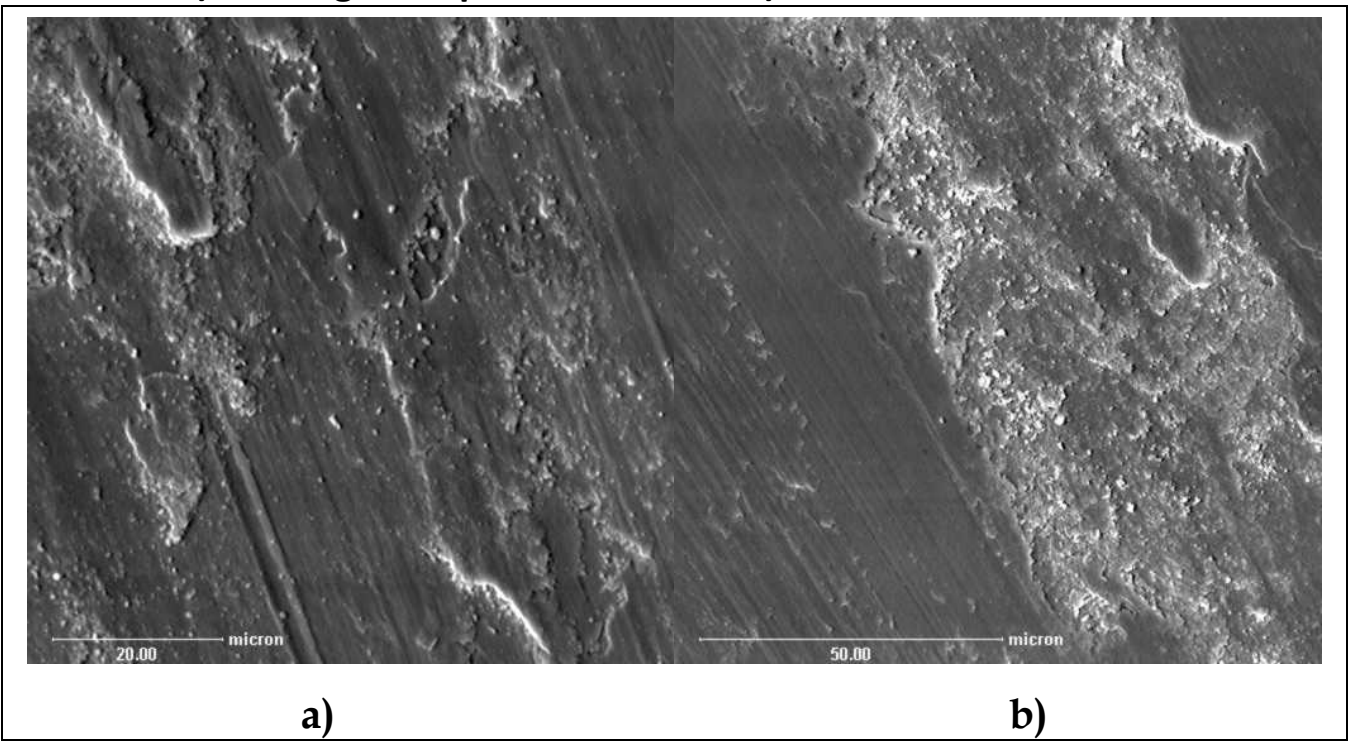

Figura 8 - Morfologia das superfícies analisadas em um SEM.

a) Laminas trincadas de óxidos. b) Lamina de óxidos globulares.

\section{CONCLUSÕES}

Podemos concluir que as variações das médias e dos desvios-padrões do coeficiente de atrito e do desgaste, contem as informações do processo tribológico que acontecem nas superfícies de contatos, podendo isolar os valores dos fenômenos que acontecem nos tribossistemas.

Os mapas de desgastes desenvolvidos nesse trabalho podem ser usados como uma ferramenta auxiliar na interpretações das regiões tribológicas dos mapas tradicionais.

Running-in ou a área de instabilidade corresponde às regiões onde os valores dos desvios padrões da taxa de desgaste e do coeficiente de atrito são maiores e as superfícies adquirem maior conformidade. 
Partindo da metodologia do estudo das flutuações dos desvios padrões dos parâmetros tribológicos, podem ser identificadas as transferências dos mecanismos de desgaste, uma vez que a variação de destes parâmetros se tornem constante.

\section{AGRADECIMENTOS}

Este trabalho foi apoiado por: CAPES (Coordenação de Aperfeiçoamento de Pessoal de Nível Superior), PRONEX (Programa de Apoio aos Núcleo de Excelência), e as empresas onde por cortesia foram realizados diferentes trabalhos, BRASIMET apoio os tratamentos térmicos e Aços Villares Sidenor pelas analises químicas das amostras.

\section{REFERÊNCIAS}

1. ARNOV, V. SOUZA, A.F.D. KALPAKJIAN, S AND SHAREEF, I. Interactions among friction wear and system stiffness - Part 2, Vibrations by dry friction, ASME, Journal of Tribology. $n 16$, , $p$ 59-64, 1984.

2. BLAU., P.J. Fifty year of research on the wear of metal. Tribology International. v30, n5, p328-331, 1997.

3. CALLACOT, R.A. Machine fault diagnosis and condition monitoring. Chapman and Hall, 1977.

4. KRISHNA, K AND SWARNAMANI, S. Vibration monitoring sliding wear of plasma sprayed ceramics, Wear. n210, p 255-262, 1997.

5. LIM, S.C. AND ASHBY, M.F. Wear Mechanism Maps, Acta Metall. 35, 1, p. 1-24, 1987.

6. PANTALEON, E.M. Modelo de desgate oxidativo baseado em parâmetros termodinâmicos. São Paulo, 2004. Teses de Doutorado - Escola Politecnica - Universidade São Paulo, 2004.

7. PANTALEON, E.M AND DOS SANTOS LIMA, G. Z. Analysis of wavelet transform applied in frictional coefficient signal. Natal, 2011. Proceedings of COBEM, CD Published. $21^{\text {st }}$ Brazilian Congress of Mechanical Engineering October 24-28, 2011, Natal, RN, Brazil

8. PEZDIRNIK, J., VIŽINTIN, J. AND PODGORNIK, B. Temperature at the interface and inside and oscillatory sliding microcontact- theoretical part. Tribology International. n32, p481489, 1999.

9. QUINN, T.F.J. Role of oxidation in the mild wear of steel. J. Appl Phys.13, p 33-37, 1962

10. QUINN, T.F.J. Oxidational Wear, ASM Handbook, vol 18, p. 280, 1992.

11. SO, H. The mechanism of oxidational wear, Wear. n184, p161-167, 1995.

12. TERCHECI, M. MANORY, R .R AND HENSLER, J.H. The friction and wear automotive Grey cast iron under dry sliding conditions. Part 1 Relationships between wear loss and testing parameters. Wear. n180, p 73-78, 1995.

13. VOGEL, A. Análise Química Quantitativa. 6 ed. Rio de Janeiro: LTC-Livros Técnicos e Científicos, 2002. 